\title{
Specification and control of screen colour in high- resolution monitors
}

\section{Document Version}

Accepted author manuscript

Link to publication record in Manchester Research Explorer

\section{Citation for published version (APA):}

Oulton, D., \& Hawkyard, C. J. (1990). Specification and control of screen colour in high-resolution monitors.

Ophthalmic and Physiological Optics, 10(3), 312.

\section{Published in:}

Ophthalmic and Physiological Optics

\section{Citing this paper}

Please note that where the full-text provided on Manchester Research Explorer is the Author Accepted Manuscript or Proof version this may differ from the final Published version. If citing, it is advised that you check and use the publisher's definitive version.

\section{General rights}

Copyright and moral rights for the publications made accessible in the Research Explorer are retained by the authors and/or other copyright owners and it is a condition of accessing publications that users recognise and abide by the legal requirements associated with these rights.

\section{Takedown policy}

If you believe that this document breaches copyright please refer to the University of Manchester's Takedown Procedures [http://man.ac.uk/04Y6Bo] or contact uml.scholarlycommunications@manchester.ac.uk providing relevant details, so we can investigate your claim.

\section{OPEN ACCESS}




\section{Abstracts}

\section{Papers presented at The Annual Meeting of the Applied Vision Association 'Human Factors in Vision', University of Manchester, 2-4 April 1990}

\section{Information presentation}

Military cockpit information displays: 'never mind the width feel the quality'

\section{Fuchs}

Sowerby Research Centre, British Aerospace PLC, PO Box 5, Filton, Bristol, BS12 7QW, UK

The proliferation in information and sensor technology has resulted in a dramatic increase in the amount of information available to the military pilot. The operator's visual system will still be the predominant human sense in future cockpits, although other modalities are being investigated; for example, direct voice input and direct touch data manipulation.

The system-display loop of display-perceive-decide-act, has to meet operational specifications, such as the pilot will be able to designate a target correctly within $3 \mathrm{~s}$. Emerging technologies have allowed the creation of innovative displays that aim to meet these ever-decreasing temporal specifications. Possible solutions to the problem of effective information transfer include increasing the number of display surfaces, the use of colour, the use of pictorial representations of tactical situations and internal systems both in two and three dimensions, and the use of 'virtual" technology.

The current state of the art in display types and formats is described: whether the solutions being provided to enhance pilot performance are meeting the requirements are discussed.

\section{The use of rapid serial visual presentation in a dual task}

\section{K. Carr and D. Higgins}

Sowerby Research Centre, British Aerospace PLC, PO Box 5, Filton, Bristol, BS12 7QW, UK

A number of items of visual information may be presented to an observer either simultaneously at different locations on a display, or in series at the same location. Matin and Boff ${ }^{1}$ have shown that serial presentations can result in more rapid information assimilation than parallel presentation. The present experiment examined the effectiveness of rapid serial visual presentation during a continuous compensatory tracking task.

The items of information used were four alphanumeric symbols, and the subjects were asked to search these for target symbols. Targets were specified before each trial, and were either consistently the same symbol, or were randomly selected from the symbol set. The symbols were presented either simultaneousely or in series. Throughout all tests the subjects were required to maintain a pivoting bar in a horizontal orientation. The results showed that there was a significantly faster response time for serial presentations, and that fewer targets were missed (there were no differences in false alarms). No significant differences were observed between the consistent and the varied targets. In addition, no effeets on the performance of the compensatory tracking task were found.

These results indicate that there may be an advantage in presenting certain types of information in a "rapid serial" format Further studies will look at the application of this technique to real tasks. One such task might be to present range or target data in series to a military pilot, at his point of gaze.

\section{Reference}

1. Matin, E. and Boff, K. R. Human Factors 30, 171-180 (1988).
Visual science and the user interface: what should go where?

\section{J. M. Findlay and D. Scott}

Department of Psychology, University of Durham, South Road, Durham, DH1 3LE, UK

Developments in the computer interface now allow almost unlimited possibilities for the presentation of material on visual display screens. Modern interfaces are designed to allow users to switch frequently between tasks or sub-tasks. Such interfaces are characterized by increasing visual complexity and increasing need to consider the characteristics of human vision and visual attention. We illustrate this by considering one particular problem: the need to provide status information to remind the user what is the current state of the system. An example of such information is whether a word processor is in insert' or 'overtype' mode. We have carried out an experiment to compare two ways of presenting such information: first, with a conventional status bar, and second, by cursor modification.

A timed record was obtained of the keystroke activity during the subjects' interactions using a specially designed monitoring system (HIMS). We compared the performance of two groups using the two different presentation methods. Although the status bar presentation was more familiar to all the subjects, the group with the cursor change presentation showed significantly faster overall performance and in particular faster mode change transitions. Prospects for developing this approach will be discussed.

The effect of pixel jitter on target acquisition from visual displays

\section{S. J. Selcon and A. J. McClumpha}

Psychology Division, RAF Institute of Aviation Medicine, Farnborough, Hants, GU14 6SZ, UK

This paper describes four studies that used objective and subjective assessments to quantify the effect of target degradation on observers" recognition ability. 'Noise' inherent in a digital infra-red (IR) line-scan system can result in a static. sinusoidal line-to-line variation (pixel jitter) over the displayed imagery. It was found that the amount of target degradation, and hence target acquisition performance, was dependent upon both the amplitude and frequency of the pixel jitter. The performance measures taken showed that, if an image is affected by pixel jitter with an amplitude of only $+/-1$ pixel, a significant interference in target recognition performance occurs $(P<0.01)$. An increasing performance decrement was found with increasing amplitudes of jitter. It was also found that the shorter the wavelength of the sinusoidal jitter waveform, the greater the performance decrement that was incurred $(P<0.01)$. The results from the subjective scaling, using a scale derived from the Cooper-Harper rating scale, mirrored closely the performance data and therefore imply that this rating scale may have widespread utility in target acquisition studies. The effect of pixel jitter appeared to be robust. These effects were found not to be specific to a particular type of imagery (both infra-red and shaded line drawings were used) and are, therefore, likely to generalize to other types of target and other imaging systems. These results are discussed in terms of existing theories of visual target recognition, and their implications for designers of visual displays are considered. 


\section{Specification and control of screen colour in high-resolution monitors}

\section{P. Oulton and C. J. Hawkyard}

\section{Department of Textiles, UMIST, PO Box 88, Manchester,} M60 1QD, UK

To carry out research in many areas of vision science it is necessary to have a means of reproducing coloured stimuli accurately. The highresolution colour CRT monitor provides an excellent basis for this, but it is not easy to ensure that the stimulus is of exactly the correct specification. To achieve a wide range of precise stimuli, a method is needed for measuring CIE coordinates of on-screen colour. The Minolta TV Analyser provides a rapid and effective method. As it is a tristimulus instrument it may be necessary to check accuracy against a full spectrophotometer, such as the Bentham telespectroradiometer (TSR). In general the Minolta achieves deviations from absolute of within +-0.005 or better in 1931 CIE two-degree $x y$ coordinates over a full range of colours.

The second important component in delivering accurate on-screen colour is a numerical method for converting $x y z$ coordinates to monitor drive ( $R G B$ ) red, green and blue values. The process involves two stages. In the first, $x y z$ coordinates are transformed into RGB coordinates using a matrix transposition. The second stage involves compensation for the non-linear relationship between drive voltage and screen luminance.

Our research has shown that these excitation curves can be measured very precisely. If the curves for each gun are measured and balanced, it is possible to reproduce colours on screen from input CIE $x y z$ to $+/-0.005$ in $x$ and $y$, and $+/-1 \%$ in luminance over the full gamut of the monitor. This will give the vision scientist a theoretical 16 million test stimuli to exact specification (that is, 256 steps on each of the R, G and $B$ scales).

There are numerous factors that may reduce the accuracy of the reproduced colour. Electronic and mechanical changes in the monitor may cause significant deviation, as does phosphor ageing. In particular, it is very important to check the gun balance regularly and avoid use during the first $30 \mathrm{~min}$ of warm-up. A second major source of inaccuracy is screen shading. Even with a monitor that looks uniform across the screen, deviations of $+1-0.02$ in $x y$ coordinates are found. In most high-resolution monitors this figure can be substantially reduced by careful adjustment of convergence (that is, the precise alignment of red, green and blue guns).

During our research we have found variations in colour with time. These may be divided into three groups: short-, medium- and long-term. While chromaticity is fairly constant over short periods, luminosity is not. Second-by-second variation of $+/-0.2 \mathrm{~cd} \mathrm{~m}^{-2}$ at a screen luminance of $20 \mathrm{~cd} \mathrm{~m}^{-2}$ was common in all our measurements. Over a period of hours both chromaticity and luminance vary significantly. From switch-on, change is rapid and substantial for the first $30 \mathrm{~min}$. The individual guns warm up at different rates. Thereafter luminance rises slowly for up to $1 \mathrm{~h}$, then drops slowly, with small changes in chromaticity of the order of $+/-0.01$ in $x$ and $y$. If the monitor remains unadjusted there are long-term variations in both gun balance and phosphor efficiency, which significantly affect colour reproduction.

To handle both short- and long-term variations in monitor colour, we have developed a system of measurement, feedback and control of colour, for calibrating colour monitors and maintaining constant colour. It can be used in learn mode to set up the necessary transposition matrix and excitation curves for any given high-resolution monitor. Subsequently a calibration mode is used to bring screen colour back to precise specification. As the latter routine is accomplished in under 2 min, it provides a convenient method of assuring on-screen colour before critical experiments.

It must be emphasized that, in our experience, it is necessary to recalibrate the monitor regularly to reproduce colour specifications accurately. It is insufficient to rely on a once-and-for-all-time initial set-up for the monitor, no matter how accurate the initial calibration was.

It is often the case that a strictly limited set of precise colours is needed. In such cases, rather than attempting to calibrate the whole colour gamut of the monitor, it is a feasible alternative to adjust individual colours. The correct $x y z$ specification is selected, the screen colour is measured, and the RGB monitor inputs fine-tuned until the screen is correct. Software can be written to allow colour specification input to be modified by small increments in either $x y z$ or RGB. In conjunction with a suitable colour-measuring instrument, the exact screen colour can be adjusted to three decimal places in chromaticity and lightness.

\section{Reading from colour displays: a psychophysical model}

\author{
D. S. Travis, S. Bowles, J. Seton and R. Peppe
}

Visual Psychophysics Laboratory, Industrial and Human Factors Design Division, British Telecom Research Laboratories, Martlesham Heath, Ipswich, IP5 7RE, UK

We have investigated the effects of chromatic and luminance contrast on reading coloured text on multi-colour displays. Stimuli were defined in a colour space with three cardinal axes. Two of these axes are chromatic and maximally modulate the opponent mechanisms of colour vision. The third axis is a luminance axis. We measured detection and discrimination thresholds for words and non-words presented in the centre of a white display. Thresholds were computed by fitting the measured psychometric curves with a Quick function. The results show that: (i) near-perfect reading at equiluminance is possible; (ii) in terms of machine units (gamma-corrected dac values), luminance differences are more salient than purely chromatic differences; but (iii) these differences disappear when the discrimination data are scaled for detection threshold.

Because of chromatic aberration and chromostereopsis, some highly saturated colour pairs are poor choices as text and background combinations. With this qualification, our results show that any combination is suitable so long as the combination maintains adequate luminance and/or chromatic contrast, where contrast is defined as a multiple of detection threshold. Perturbations in these channels are discriminated if the contrast in the channel is greater than or equal to a contrast $k$, where:

$$
k=\frac{\text { discrimination threshold }}{\text { detection threshold }}
$$

In our experiments, $1.23<k<2.09$, and there is no significant difference in the values of $k$ between the luminance and chromatic channels. However, since large multiples of contrast threshold are easier to realize in the luminance channel than in the chromatic channels, we suggest colour pairs are used that maintain a luminance contrast modulation of $50 \%$. This accords with the current recommendation in the ISO Draft International Standard $(9241 / 3)$

\section{Tests of a new definition of colour constancy}

\section{B. J. Craven, D. H. Foster and E. R. H. Sale}

Department of Communication and Neuroscience, University of Keele, Keele, Staffordshire, ST5 5BG, UK

Conventional definitions of colour constancy imply that a given reflecting surface should, to a perfectly colour-constant observer, appear identical under a range of different illuminants. Published experimental data fail to show such behaviour in human subjects. We propose that the conventional definition of colour constancy may be too strict, and that colour constancy might be better considered as the ability of an observer to determine correctly whether a change in the appearance of a surface is due to a change in the reflecting properties of the surface or to a change in the incident light. We assessed this ability using a computer simulation of patterns of Munsell chips illuminated by different phases of daylight. In a 2-AFC design, subjects were required to decide which of two patterns could be derived from a third by a change in illuminant without a change in material. We measured performance as a function of the number of elements in the displays and the stimulus duration. Pilot experiments show that subjects are more successful as the number of elements in the stimulus patterns is increased, and can in some cases perform well above chance when the stimuli are presented for a single refresh cycle of the display.

\section{The VDU environment}

\section{Visual fatigue caused by sustained near work}

\section{L. Ehrlich}

Department of Optometry and Vision Sciences, UMIST, PO Box 88, Manchester, M60 1QD, UK

The ability to focus beyond the resting state ('dark focus') towards the true cycloplegic far point was compared in hyperopic and myopic 
students. Hyperopes, who are 'habitual accommodators', normally accommodate for their hyperopia, which maintains a latent 'tonus'. This acts to prevent them reaching their far point. Myopes have no habitual far point tonus. The hyperopes' far response was significantly more variable and was related to their dark focus as well as to the magnitude of their hyperopia.

'Temporary accommodators' were created by making subjects do a $2 \mathrm{~h}$ experimental fine near task at $20 \mathrm{~cm}$ without any breaks. These 'tempora"y accommodators', as well as the 'habitual accommodators', showed similar difficulty focusing towards their far points.

These results indicate that continuous sustained accommodation makes it difficult for subjects to relax their accommodation from their dark focus towards their far point. Good visual ergonomics that reduce near visual demands, such as by increasing working distances and the taking of frequent breaks, should prevent these problems.

\section{The perception of flicker on raster-scanned displays}

\section{W. D. Thomson and J. Saunders}

Department of Optometry and Visual Science, The City University, Dame Alice Owen Building, 311-321 Goswell Road, London, EC1V 7DD, UK

A raster-scanned display produces a complex pattern of spatiotemporal modulation. The perception of flicker produced by such a stimulus cannot be predicted accurately by conventional models describing modulation of a spatially uniform field. In addition to the range of variables influencing the perception of flicker in a spatially uniform field, the nature of the flicker perceived on a raster-scanned display depends on a number of other factors. For example, eye movements in the direction of the field scan produce a brief burst of flicker due to the reduction in the relative velocity between the eyes and the field scan. Data will be presented that show that for a given field scan frequency (in the region of the flicker fusion frequency) flicker becomes more perceptible if the field scan direction is changed from the conventional top to bottom to either bottom to top, left to right or right to left. A correlation was found between TV/VDU viewing history and the extent of this asymmetry, suggesting a long-term adaptation effect to raster-scanned displays. The implications of these findings for the design of raster-scanned displays will be discussed.

\section{Accommodative response to flickering stimuli}

\section{W. N. Charman and K. Chauhan}

Department of Optometry and Vision Sciences, UMIST, PO Box 88, Manchester, M60 1QD, UK

\section{A. Halnan, C. Kelly, A. Loughlin, K. Neilson and G. Walsh \\ Department of Ophthalmic Optics, Glasgow College, Cowcaddens Road, Glasgow, G4 0BA, UK}

It has recently been suggested ${ }^{1}$ that accommodative accuracy may be influenced by flicker at rates above the critical fusion frequency (CFF). A study has been performed to test the validity of this. Such a result would be of considerable significance in relation to VDU use and to work under fluorescent lighting systems.

An LED matrix in a Badal optical system was used enabling flicker rates and waveform to be accurately maintained whilst keeping angular subtense constant at levels of accommodative demand up to $6 \mathrm{D}$. Accommodation was monitored using a Cannon open view optometer.

Results to date show no systematic variation in accommodation accuracy with near- and above-CFF flicker.

\section{Reference}

1. Neary, C. The effect of high frequency flicker on accommodation. Ophthal. Physiol. Opt. 9, 440-446 (1989).

\section{Open contributions}

Compensatory head postures and congenital nystagmus

\section{R. V. Abadi and J. Whittle}

Department of Optometry and Vision Sciences, UMIST, PO Box 88, Manchester, M60 1QD, UK

Individuals with congenital nystagmus frequently adopt an abnorma head posture whenever there arises the need to resolve fine detail The purpose of this study was to investigate which factors determined the nature and extent of the head posture. The head positions and eye movements of 16 subjects were monitored whilst they took up a variety of gaze positions. Five subjects displayed a single head posture and four showed multiple head postures. Six of the nine head postures matched with the minimum intensity zone. The extent of the head posture was also found to be dictated by the velocity distribution of the slow phase, the nystagmus beat direction and the position of the waveform reversal zone. These results suggest that the surgical management of the head posture should not always be based on the relocation of the minimum intensity zone to the primary position of gaze.

The influence of spatial distortion products on perceived speed

\section{G. K. Edgar and A. T. Smith}

School of Psychology, University of Wales College of Cardiff, PO Box 901, Cardiff, CF1 3YG, UK

A complex grating pattern comprising two sine components has the appearance of a single contrast-modulated grating, the period of the contrast modulation being equal to the difference between the two components. When such a pattern, comprising two sine components of 14 and $16 \mathrm{c} / \mathrm{deg}$, was drifted across the screen the high spatial frequency components became invisible at speeds greater than about $3 \operatorname{deg~s}^{-1}$

Between approximately 3 and $6 \mathrm{deg} \mathrm{s}^{-1}$, however, an illusory grating was visible in place of the now invisible high spatial frequency complex grating. We matched a real grating to an illusory grating elicited by the complex grating drifting at $3.8 \mathrm{deg} \mathrm{s}^{-1}$. The illusory grating was of low contrast (about 0.012 ) and appeared to drift at about the velocity of the inducing pattern. It appeared to be sinusoidal and its perceived spatial frequency was similar to the difference frequency of the inducing pattern. The illusory grating may arise from a brightness non-linearity, possibly in the lateral geniculate nucleus. Such a non-linearity could give rise to a distortion product which, because of its relatively low spatial frequency, survives the spatio-temporal filtering imposed by the visual cortex even though the original pattern does not. Further experiments using a variety of complex gratings (e.g. missing-fundamental) suggest that the presence of distortion products may affect the perceived speed of these gratings, even though the distortion products themselves may not be visible.

\section{Contrast sensitivity in the periphery as a function of colour, viewing angle and field size}

\section{P. Parsley and J. R. Wilson}

Department of Production Engineering, University of Nottingham, University Park, Nottingham, NG7 2RD, UK

One significant problem in cockpit design is the location of displays and controls which must transfer important information to the pilot on his peripheral visual field. To investigate this area and provide reference guidelines to design engineers a series of experiments were run using two subjects. Contrast sensitivity functions for chromatic and achromatic Gabor gratings were obtained at retinal eccentricities of $0^{\circ}$, $15^{\circ}, 30^{\circ}, 40^{\circ}$ and $50^{\circ}$ on the horizontal temporal meridian. Three circular field sizes were used, subtending angles of $10^{\circ}, 6.7^{\circ}$ and $3.4^{\circ}$. Spatial frequencies of the gratings were $12,6,3$ and $1.5 \mathrm{c}$ /deg of visual angle. Field colours equated to the three primary CRT colours (red, 
green and blue) and a white field was also used. Ambient illumination was set at 1100 lux to simulate daylight cockpit conditions. It was found that:

1. High ambient illuminant conditions shift one's peak contrast sensitivity from $3 \mathrm{c} / \mathrm{deg}$ to approximately $6 \mathrm{c} / \mathrm{deg}$ when viewed at an angle of 0

2. There is no significant difference among colour response in the periphery (up to $50^{\circ}$ ) or the fovea.

3. Peripheral contrast sensitivity reduces by a factor of 10 over the range of $0^{\circ}$ to $50^{\circ}$.

4. There is a linear relationship between field size and contrast sensitivity in the periphery.

\section{Visual function in demyelinating disease}

M. H. A. Russell and I. J. Murray

Visual Sciences Laboratory, UMIST, PO Box 88, Manchester, M60 1QD, UK

\section{R. A. Metcalfe and J. J. Kulikowski}

Department of Neurology, Manchester Royal Infirmary, Oxford Road, Manchester, UK

The majority of multiple sclerosis (MS) patients have an episode of optic neuritis during the course of the disease. The observation that this is often associated with an acquired colour vision defect has prompted a number of psychophysical investigations of selective damage to colour or luminance pathways. These investigations have reached apparently conflicting conclusions. To clarify the nature of the visual pathology in MS and optic neuritis we have used psychophysical and electrophysiological techniques to stimulate selectively chromatic and achromatic mechanisms in MS patients and in patients recovering from an episode of optic neuritis. The onset of a low contrast, low spatial frequency isoluminant red-green grating at a low repetition rate $(1 \mathrm{~Hz})$ was used to obtain visually evoked potentials (VEPs), which selectively activate the chromatic system. Isoluminance was determined using heterochromatic flicker photometry. Low-contrast achromatic gratings and checkerboard stimuli were used to record achromatic VEPs. VEP amplitudes and latencies were compared with $1 \mathrm{~Hz}$ and $25 \mathrm{~Hz}$ spectral sensitivities. In normals, $1 \mathrm{~Hz}$ and $25 \mathrm{~Hz}$ spectral sensitivities have been shown to monitor the chromatic and achromatic mechanisms respectively.

Thirty-two patients with MS were investigated ( 15 definite, 8 probable and 11 possible). None of the patients was having a clinical relapse of visual symptoms at the time of the examination. Damage to colour and luminance pathways appeared to be equal, although in some cases high temporal frequency sensitivity was greatly depressed. There was a significant correlation between $1 \mathrm{~Hz}$ spectral sensitivity and chromatic VEP amplitude, indicating that the latter may prove to be useful for objectively monitoring colour vision.

A second group consisting of 12 patients with acute optic neuritis was assessed periodically until vision stabilized. During the acute phase, $25 \mathrm{~Hz}$ spectral sensitivity was more severely affected than $1 \mathrm{~Hz}$, which was selectively depressed at short wavelengths. After recovery, results were as for the MS patients with normal $25 \mathrm{~Hz}$ spectral sensitivity and a small residual defect at $1 \mathrm{~Hz}$, which was uniform across the spectrum.

\section{Visual impairment}

\section{Assessing the effects of differing cataract morphologies on visual function}

\author{
M. A. Hurst, D. B. Elliott and J. R. Weatherill
}

Clinical Vision Research Unit, Department of Optometry, University of Bradford, Bradford, W. Yorks, BD7 1DP, UK

Cataract patients have a decreased visual function due to increased lenticular light scatter, which reduces the contrast of the retinal image. Conventional techniques for assessing the visual function of cataract patients include visual acuity, contrast sensitivity and glare disability. These measurements were made on 79 subjects having normal retinal function, using commercially available techniques. Of these, 43 had cataracts of a pure morphological type, 11 had cataracts of a mixed morphology and 25 had clear media. Visual acuities of all subjects were better than equivalent Snellen 6/18. Each subject's crystalline lens was evaluated using the Oxford Clinical Cataract Classification and Grading System. Visual acuity was measured using a Ferris logMAR chart and contrast sensitivity by the Pelli-Robson letter chart. Glare disability was evaluated using the Mentor Brightness Acuity Tester in conjunction with both the $\log$ MAR and Pelli-Robson charts.

Of the pure types of cataract there were insufficient numbers of nuclear and anterior subcapsular morphologies to allow adequate statistical analysis. Both cortical, and particularly posterior subcapsular types, showed a statistically significant reduction in contrast sensitivity from normal. This was not correlated to visual acuity. There was no significant correlation between all of the glare disability and visual acuity scores for patients with both of these cataract morphologies.

The clinical significance of these results wll be discussed in relation to the pre-surgical evaluation of cataract patients.

\section{Eye movements and reading in age-related maculopathy}

\section{A. Bullimore and I. L. Bailey}

School of Optometry, University of California, Berkeley, CA 94720, USA

Age-related maculopathy (ARM) typically has a profound effect on an individual's ability to read. Reading characteristics were studied by monitoring eye movement patterns as normally sighted and ARM subjects read word charts and text charts that contained a wide range of print sizes. Eye movements were monitored with an infra-red scleral reflection device and subjects were tested over a wide range of chart luminances.

In normally sighted subjects reading slows as print size approaches threshold. This was found to be due to a decrease in fixation rate. We found the ratio of number of letters read to the number of forward saccades to be almost constant and regressive saccades were rare.

ARM subjects read slowly for all print sizes. Their slower reading is most strongly associated with a decrease in the average number of letters per forward saccade and they also make more regressions. Their fixation rates are, however, remarkably similar to those of normals. ARM subjects were more likely to have their reading performance affected by luminance. When this occurred, fixation rate and regression rates were relatively unchanged, while the number of letters per forward saccade showed a strong dependence on luminance.

The influence of scotoma size and eye movement control on reading performance in ARM will be discussed, along with recent technological developments of the visually impaired.

\section{Impaired vision and reading}

\section{M. Dickinson}

Department of Optometry and Vision Sciences, UMIST, PO Box 88, Manchester, M60 1QD, UK

\section{P. M. A. Rabbitt}

Age and Cognitive Performance Research Centre, Oxford Road, Manchester, M13 9PL, UK

When the reading performance of visually impaired subjects is compared using different low-vision aids, it is common to rate the 'success' of an aid by the increased reading rate that it allows. The goal of reading is however to gather information from the text and it may be that this ability is impaired because of the low vision, regardless of the reading rate.

To investigate this possibility, normal subjects aged between 18 and 35 years were given two different texts (approximately 450 words long) to read aloud, whilst attempting to remember the content. They viewed 
one through their normal refractive correction, and the other with additional distorting lenses. The order of the two tasks was randomized. In each condition, the subjects were then tested for their free recall, and for their recognition of printed phrases from the text ('prompted recall').

The interaction of text difficulty, reading speed, subject's IQ and text recall in subjects with distorted vision was then evaluated to determine whether reading rate gives an accurate description of reading performance in such cases.

\section{Information for rehabilitating the visually handicapped}

A. R. Hill

Department of Optometry, Oxford Eye Hospital, Walton Street, Oxford, OX2 6AN, UK

\section{P. A. Aspinall}

Department of Ophthalmology, Alexandra Eye Pavilion, Edinburgh, UK

For many years there has been considerable confusion about the nature of the information that is required by those involved in rehabilitating the visually handicapped. Specialist social workers for the blind and visually handicapped claim that clinical information about the patient/ client is essential for effective decision-making about rehabilitation, while ophthalmologists assert that such information is subject to the general principle of medical confidentiality and should therefore be available only to medical colleagues.

In an attempt to solve the dilemma presented by these divergent professional views, we have conducted a study using Kelly's Repertory Grid interview technique ${ }^{1}$ to identify what information social workers actually use in making decisions about alternative courses of action for rehabilitation. Over 200 attributes of information were elicited. Principal component and cluster analysis revealed four major areas of essential information

1. Aspects of visual function.

2. Functional aspects of general health.

3. Level of support

4. Personal traits and aptitudes.

Information on clinical diagnosis was not used in decisions about rehabilitation.

Ratings of satisfaction and confidence in using scales derived from the analyses were obtained from ophthalmologists and social workers Validation of the appropriateness of the scales is currently in progress

\section{Reference}

1. Kelly, G. A. The Psychology of Personal Constructs, Vols. I and II Norton, New York (1955).

\section{Clinical ergonomics}

\section{Reliability of a computerized optic disc assessment system}

\section{J. Cox and I. C. J. Wood}

Department of Optometry and Vision Sciences, UMIST, PO Box 88, Manchester, M60 1QD, UK

Fast and reliable automated assessment of the optic nerve head should be a useful aid to diagnosis and follow-up in diseases such as openangle glaucoma. A computerized optic disc analysis system has been produced running on an IBM PC/AT compatible computer system.

The disc analysis software is used to measure linear pallor-to-disc (P-to-D) ratios, horizontally and vertically, the area pallor-to-disc ratio, and a pallor ratio that also measures the relative pallor between the pallor area and the neuro-retinal rim. Disc and pallor area eccentricity is also computed. This proces takes about $3 \mathrm{~min}$ in total.

Three subject groups (normal, ocular hypertensive and glaucomatous) each had five subjects. Each subject was photographed 10 times, and the images subsequently analysed in a randomized, masked order, to give a measure of the reliability of the system.
The coefficients of variation of these parameters are shown below.

\begin{tabular}{llll}
\hline & $\begin{array}{l}\text { Glaucomatous } \\
(\%)\end{array}$ & $\begin{array}{l}\text { Ocular } \\
\text { hypertensive } \\
(\%)\end{array}$ & $\begin{array}{l}\text { Normal } \\
(\%)\end{array}$ \\
\hline $\begin{array}{l}\text { Vertical } \\
\text { linear P-to-D }\end{array}$ & 2.46 & 4.90 & 7.52 \\
$\begin{array}{l}\text { Horizontal } \\
\text { linear P-to-D }\end{array}$ & 2.64 & 4.62 & 5.72 \\
$\begin{array}{l}\text { Area P-to-D } \\
\begin{array}{l}\text { Disc area } \\
\text { eccentricity }\end{array}\end{array}$ & 5.70 & 6.72 & 9.80 \\
$\begin{array}{l}\text { Pallor area } \\
\text { eccentricity }\end{array}$ & 1.36 & 1.66 & 1.86 \\
Pallor ratio & 8.38 & 4.56 & 6.24 \\
\hline
\end{tabular}

These results shows that the system has a reliability in line with that of other nerve head analysis systems, but it uses a monoscopic system, and has the advantage of real-time processing.

\section{Design guidelines for an image-based medical work-station}

G. von Voigt

Department of Computer Science, University College London, Gower Street, London, WC1E 6BT, UK

In the last few years, the number and variety of digital imaging systems in radiological departments has increased so that more computers have moved into hospitals. Therefore, computing power now exists and people are beginning to become familiar with its use. Part of the patient data is created by digital imaging systems and thus it is available in a digital format. It seems obvious to the author to utilize these circumstances for other tasks, for example:

1. To give assistance to the radiologist and/or treating physician in finding the diagnosis.

2. To help in the planning of the treatment and to enable the clinician to simulate the patient's treatment.

3. To make the administration of the patient data easier.

This requires that the interface for a medical work-station, which should incorporate the above-mentioned tasks, takes care of the special circumstances found in a hospital and is centred around the particular user-groups, such as radiologists and/or clinicians.

The purpose of this paper is, first, to give main guidelines for the whole design process to the designer of a medical work-station and second, to present the rules for a good interface design. The main guidelines for the design process of an interface for a medical workstation are based on the idea of user-centred design ${ }^{1}$. This requires the developer to fulfil the following steps:

1. Understand the user. Evaluate the goals, benefits and costs of the physician, surgeon, radjologist, radiographer, hospital administration and management. Describe and characterize these users and gather the product requirements from that.

2. Understand the user's tasks. Characterize the tasks of the physician, surgeon, radiologist, radiographer, hospital administration and management and deduce product requirements. Investigate the working conditions and note if they require special hardware or software.

3. Specify the evaluation of the existing design. Define the goals that the medical work-station has to meet; find a procedure with which you can test this goal; decide how you want to measure the test what are the desired and the minimal acceptable levels of this measurement; find an appropriate data collection method.

4. Design the user interface. Decide which style of interaction is the most appropriate to the medical environment; give special attention to the provision of help facilities; consider the requirements for an interface that has to deal with two- and three-dimensional images.

5. Implement a prototype. The test of the system's design with a prototype avoids an expensive and unsuccessful product development 
6. Redesign or product development. If the test fails, the interface has to be redesigned; if the test is satisfactory, the interface for the medical work-station can be developed.

A good interface should be understood as a user-friendly one and has to be easy to handle, self-explanatory, quick to use and should incorporate the whole patient folder.

\section{Reference}

1. HUSAT. Tools for User Centred Design. HUFIT Seminar, Loughborough University of Technology, UK, October 10-13 (1989).

\section{Using standard PC monitors to measure acuity and screen the visual fields}

\section{N. French, N. Oldbury and I. C. J. Wood}

Department of Optometry and Vision Sciences, UMIST, PO Box 88, Manchester, M60 1QD, UK

\section{R. McNamara}

Orthoptics Department, Manchester Royal Eye Hospital, Oxford Road, Manchester, M13 9WH, UK

A short clinical trial in an orthoptic clinic of a computer program that simulates STYCAR single-letter test procedures (and in addition allows letters to be presented surrounded by crowding bars) is reported. The test's results are compared with those from the conventional SheridianGardiner single-letter test and Snellen chart, and its acceptance by children is assessed.

A computer algorithm is described that automatically controls the presentation of visual field targets and analyses the subject's responses Data relating to the procedure's reliability and validity are reported along with experience in using it with normal subjects on an IBM PC compatible. It is argued that behavioural control techniques can greatly reduce the need for an operator. The limitations of standard PC monitors (CGA, Hercules and VGA) will be discussed.

\section{Retinal irradiance received by a preterm neonate}

\section{J. Robinson}

Department of Ophthalmology, University of Birmingham, Birmingham and Midland Eye Hospital, Church Street, Birmingham, B3 2NS, UK

Early exposure to bright light may affect the immature visual system and has been implicated in the pathogenesis of retinopathy of prematurit (ROP). The lighting environment in neonatal units has been studied to determine the amount of light reaching the baby's eyes.

Both physical (e.g. illuminance, spectral irradiance) and physiological (e.g. eyelid opening, eyelid transmission, pupil size) factors have been examined. Lighting surveys were performed in seven neonatal units in the Midlands, UK. Mean unit illuminance was 470 lux (range 192-890 lux). The intensive care nurseries were significantly brighter than their corresponding low-dependency nurseries. Photodiodes, placed near the eyes of 65 infants, have enabled monitoring of the illuminance actually reaching the eye or eyelid over time periods of between 4 and $24 \mathrm{~h}$ (sample interval approximately $10 \mathrm{~s}$ ). These results suggest that between $30 \%$ and $98 \%$ of the available light actually reaches the baby's eyelid or eye with least light reaching the eye nearest the mattress.

The transmission of light through the eyelid of preterm infants has been measured in vivo and our results suggest that the eyelid acts as predominantly red-pass filter, permitting $30 \%$ transmission at $700 \mathrm{~nm}$ with $<5 \%$ transmission at $420 \mathrm{~nm}$

Using all the available data, including previously reported estimates of eyelid opening frequency and pupil diameter, an estimate of the light dose received by a preterm neonate in a neonatal unit has been derived (approximately $350 \mu \mathrm{W} \mathrm{cm}^{-2}$ ). Those infants potentially at greates risk for developing severe ROP are also those who will receive the largest ocular light exposure during their stay in neonatal units.

These data will provide a basis for future studies investigating the effects of light on the growth and development of the eye and visual pathways and may help when considering the relation between light levels in neonatal units and the incidence of ROP and other visua pathway defects.

\section{Human factors in $\mathbf{R} \& \mathbf{D}$}

\section{The design of helmet-mounted displays}

\section{K. T. Carr}

Sowerby Research Centre, British Aerospace PLC, PO Box 5, Filton, Bristol, BS12 7QW, UK

A number of helmet-mounted displays (HMDs) have been developed recently, mostly for use in teleoperation, military cockpits and entertainment. The imagery presented on the HMD is linked to head movement, so that an unlimited total field of view is possible, allowing complete 'virtual' environments. HMD design has been determined largely by the available technology, in particular CRT or LCD displays and optics. As engineers strive to improve that technology, human factors researchers must determine what the goals of that technology must be. For example, a wide instantaneous field of view is clearly desirable, but just how wide should it be? What are the costs and benefits for every increase in the HMD's field of view? The ideal specifications for a HMD depend upon its application, but a useful starting point for research is to investigate a number of basic visual tasks with a HMD.

This paper will report the effects of differing binocular overlap in a simple visual search task. Two eyepieces of 60-degree visual angle were each arranged variously to produce no binocular overlap, a small overlap of 10 degrees, an overlap of 30 degrees, and total overlap. The search field was 60 degrees wide and consisted of simple geometric shapes. Search performance was measured in each condition and a subjective evaluation obtained from each subject. The results will be presented, and implications for HMD design discussed.

\section{Indicating switch selection with head position}

\section{T. Simpson}

Sowerby Research Centre, British Aerospace PLC, PO Box 5, Filton, Bristol, BS12 7QW, UK

The position of the head can be used to select switches. Pointing the head at a switch would provide a useful alternative to finger switching in a range of applications, such as aerospace and assisting disabled people. The aim of this experiment was to determine the speed and accuracy of head pointing at targets. The method used a visual display unit directly in front of the subject on which targets and a headcontrolled reticle were drawn. Targets were drawn off-axis in a range of directions. The results in terms of time and errors are reported for a range of target sizes.

\section{Human factors in map-user performance}

\section{A. R. Hill}

Department of Optometry, Oxford Eye Hospital, Walton Street, Oxford, OX2 6AN, UK

The evaluation of most map products is based upon map-room tasks, which measure the accuracy of interpretation of the cartographic symbol key. It is of particular practical interest to know how well a map-user can visualize the type of terrain from a map and how readily objects in the field can be correlated with their symbolic representations on the map. Map reading performance has been studied both in a map-room and in the field, as a means of comparing the efficiency of alternative reprographic forms and cartographic treatments of map production.

The experiments examined map-user performance at a fixed location in the field to which the subject had been taken in a closed vehicle. A striking feature of the results was the extent of the individual differences in performance, given that the subjects all held qualifications in navigation. Despite the inclusion in the experimental design of radically different types of map, ranging from completely new products to the traditional Ordnance Survey line map, these differences accounted for only a small proportion of the variation in map-user performance Highlighting selected linear features (e.g. roads, railways and waterways) produced a significant improvement in performance, independent of reprographic form. 